AperTO - Archivio Istituzionale Open Access dell'Università di Torino

\title{
Clinical evaluation of an end-tidal target-controlled infusion closed-loop system for isoflurane administration in horses undergoing surgical procedures
}

\section{This is the author's manuscript}

Original Citation:

Availability:

This version is available http://hdl.handle.net/2318/1691475

since 2019-02-09T18:15:04Z

Published version:

DOI:10.1016/j.tvjl.2011.06.001

Terms of use:

Open Access

Anyone can freely access the full text of works made available as "Open Access". Works made available under a Creative Commons license can be used according to the terms and conditions of said license. Use of all other works requires consent of the right holder (author or publisher) if not exempted from copyright protection by the applicable law. 


\section{Title page}

Comparison of two isoflurane delivery systems for maintaining anaesthesia in horses: end-tidal closed-loop target-controlled vs out-of-circuit vaporizer

S. Levorato, P. Franci, B. Contiero and R. Busetto

Department of Animal Medicine, Productions and Health, University of Padua, Via dell’Università, 16 Agripolis - 35020 Legnaro (PD) - Italy

silvy_vet@hotmail.it

barbara.contiero@unipd.it

roberto.busetto@unipd.it

Correspondence: Paolo Franci, Via dell’Università, 16 Agripolis - 35020 Legnaro (PD) Italy

E-mail: paolo.franci@unipd.it

Tel. $+39(0) 8272951$

Fax $+39(0) 8272954$ 


\section{Abstract}

The purpose of this study was to compare two different isoflurane (Iso) delivery systems (i) end-tidal closed-loop target-controlled infusion (ETCLo-TCI) system and (ii) out-ofcircuit vaporizer (VOC) - in horses undergoing surgery.

Twenty-four horses undergoing scheduled surgery were enrolled in this study. Anaesthesia was maintained using Iso delivered either by a calibrated TEC 4 VOC vaporizer (group V, $\mathrm{n}=14$ ) or an ETCLo-TCI system (group $\mathrm{I}, \mathrm{n}=10$ ). Data were collected within the first hour of anaesthesia on the consumption of Iso, the number of anaesthetist drug delivery adjustments (software changes to the vaporizer setting or FE'Iso target input), the number of fresh gas flow (FGF) changes and the time taken to reach the first FE'Iso target $(1.5 \%)$ after induction.

The median consumption of Iso and the median consumption of Iso per kilogram of body weight (BW) during the first hour of anaesthesia were significantly lower in group I compared with group V (20.5 (9.3-31.4) vs $29.1(21.5-43.0)) \mathrm{mL},(P=0.003)$, and 0.042 (0.028-0.052) vs $0.062(0.037-0.094) \mathrm{mL} \mathrm{kg}^{-1},(P=0.009)$. The median number of anaesthetist drug delivery adjustments was significantly lower in group I compared with group V (1.5 (0-3) vs $4(3-9)),(P=0.0002)$.

The focus was on the number of drug delivery adjustments required to reach and maintain the target end-tidal Iso concentration (FE'Iso) and Iso consumption within the study period. The time taken by both delivery systems to reach the FE'Iso of $1.5 \%$ as first target was also considered. 
Keywords: horse; isoflurane; vaporizer; closed-loop; target controlled infusion 


\section{Introduction}

Quantitative anaesthesia is the delivery of the exact amount of anaesthetic required to maintain the desired depth of anaesthesia in the anaesthetized subject [1]. This technique has been shown to have some advantages over qualitative anaesthesia, such as easy and efficient monitoring of the uptake of anaesthetic vapours, a substantial reduction in pollution, reduced costs, and continuous measurement of $\mathrm{O}_{2}$ uptake and $\mathrm{CO}_{2}$ production [1]. Despite its reported advantages, quantitative anaesthesia using the low gas flow technique has been unpopular because there is a conflict between reducing the gap between the desired and actual drug concentration and minimizing agent consumption by decreasing the fresh gas flow (FGF), particularly when using conventional anaesthesia machines [2]. Injecting a vatile agent, in its liquid form, This conflict is resolved when the volatile anaesthetic agent is administered by injecting it directly into the respiratory circle system [3]. However, though satisfactory results were obtained when quantitative anaesthesia was attempted by manual injection of a volatile liquid anaesthetic into a closed breathing circuit according to the square root of the time, there was a failure to predict the volatile anaesthetic uptake and cardiovascular depression [4-8].

Various experimental and commercial anaesthesia delivery systems using closed-circuit technology together with in-circle agent delivery have been described in the literature, with each study attempting to identify the criteria that could be used to achieve agent control and delivery $[3,9,10]$. These computerized delivery systems should aim to allow the anaesthetist to obtain a particular concentration of anaesthetic by simply entering a value into the computer controlling the system. Target-controlled inhalational (TCI) anaesthesia 
is a method of producing anaesthesia based on controlled infusion by using an inhalational anaesthetic delivery system, where the system automatically adjusts the anaesthetic agent concentration to achieve the desired target levels set by the user. Such a system has been found to reduce anaesthetic consumption, as well as the average time required to achieve the desired FE' of the volatile agent and the number of drug delivery adjustments required to maintain anaesthesia [11].

A low-budget electronic prototype of an isoflurane (Iso) delivery system, an end-tidal closed-loop target-controlled infusion (ETCLo-TCI), was tested on horses undergoing various surgical procedures [12]. The anaesthetist had only to input the desired FE'Iso (ETtarget***) into the anaesthetic delivery system and set the FGF manually. Iso was directly injected into the breathing system by a closed-loop system, comprising a laptop computer (with dedicated software), a computer-controlled syringe driver (loaded with liquid Iso) connected to the inspiratory arm of a large-animal circle breathing system and a respiratory gas monitor providing Iso end-tidal concentrations (FE'Iso) every 20 seconds to the computer (Figure 1). This system works by administering an initial loading dose, based on Lowe's equation [13], which is simply calculated by considering the total volume of the breathing system and the ventilator bellows, the functional residual capacity and the target FE'Iso and roughly estimating the cardiac output (CO). The anaesthesia infuser-delivery system then injects Iso every minute in order to maintain the target FE'Iso. This system proved to be sufficiently accurate and easy to use [12].

The aim of this study was to assess during the first hour of inhalational anaesthesia whether the ETCLo-TCI system provides some advantages over a VOC vaporizer, in terms of the amount of Iso consumed, the number of drug delivery adjustments required and the time 
needed to reach the targeted FE'Iso in horses undergoing surgery.

\section{Materials and Methods}

The institutional Ethics Committee of the University of Padua discussed and approved the study procedure (Protocol number 69012; 2009; Tit III; Cl. 11; Fasc. 10).

Owners were informed about the ongoing nature of the study and signed the anaesthesia consent form. The estimated sample size to detect a difference in the primary endpoint (isoflurane consumption in one hour of anaesthesia) with a power of $80 \%$ and an alpha error of 5\% in a two-independent group study design was calculated using commercially available software (Statistica, Statsoft IT). The effect size calculation was obtained assuming a hypothesized mean difference of $8 \mathrm{~mL}$, with a standard deviation of $6.5 \mathrm{~mL}$. The calculated minimum sample size was 10 subjects for each group. Horses recruited for this study were undergoing isoflurane-maintained general anaesthesia for scheduled surgical procedures. Only horses positioned in dersal recumbence position dorsal recumbency were enrolled for this study.

\subsection{Anaesthetic technique}

Horses were randomly assigned to group I or group V. Subjects assigned to the former group were maintained in anaesthesia using the ETCLo-TCI system, while those assigned to group $\mathrm{V}$ were anaesthetized with an isoflurane VOC vaporizer (TEC 4; Ohmeda, Finland). It was noted on the anaesthesia record if the subject being anaesthetized was the first case of the day. The health of the horses was determined by their history and a physical examination. The physical status of the horse (American Society of Anesthesiologists (ASA) classification) was recorded, as well as its body weight. Food, but not water, was 
withheld for $10-12 \mathrm{~h}$ before anaesthesia. All horses received acepromazine $\left(0.02 \mathrm{mg} \mathrm{kg}^{-1}\right.$; Prequillan; Fatro, Italy) and phenylbutazone (4.4 $\mathrm{mg} \mathrm{kg}^{-1}$; Bute; ACME, Italy) intravenously (IV), via a previously inserted $12 \mathrm{G}$ jugular catheter, 30 minutes before induction. This was followed by the administration of either romifidine $\left(0.08 \mathrm{mg} \mathrm{kg}^{-1}\right.$; Sedivet, Boehringer Ingelheim, Germany) or xylazine (1 $\mathrm{mg} \mathrm{kg}^{-1}$; Sedaxylan $2 \%$; Vetem Eurovet Animal Health, United Kingdom). Ketamine (2.2 $\mathrm{mg} \mathrm{kg}^{-1}$; Ketavet 100, Intervet Productions, Italy) and diazepam (0.060 $\mathrm{m} \mathrm{kg}^{-1}$; Diazepam Hospira, Hospira Italia, Italy) were used to induce anaesthesia in all of the horses. After orotracheal intubation, the cuff was inflated and the horse was connected to a large-animal breathing system (Rotatorio Grossi Animali; SAMED, Italy). The horse was then placed in a dorsal recumbency on the operating table.

The breathing system comprised a standard large-animal circle anaesthetic system that included an integrated ventilator with descending bellows (Ventilatore Grossi Animali; SAMED, Italy). In order to detect potential leakages the system was filled with oxygenuntil a pressure of $25 \mathrm{~cm} \mathrm{H}_{2} \mathrm{O}$. In order to detect potential leakages, the system was pressure checked at $25 \mathrm{cmH}_{2} \mathrm{O}$.

To maintain the end-tidal carbon dioxide concentration $\left(\mathrm{FE}^{\prime} \mathrm{CO}_{2}\right)$ below $5.2 \mathrm{kPa}$ and to guarantee a more stable and predictable Iso uptake, positive-pressure ventilation was immediately started at a peak inspiratory pressure of $20 \mathrm{cmH}_{2} \mathrm{O}$, with a respiratory frequency ranging between 7 and 8 breaths per minute. A respiratory gas monitor (Cardiocap Ultima; Datex-Ohmeda, Finland) was used to continuously measure the following respiratory variables: inspired $\left(\mathrm{FI}^{\prime} \mathrm{O}_{2}\right)$ and expired $\left(\mathrm{FE}^{\prime} \mathrm{O}_{2}\right)$ oxygen fractions, $\mathrm{FE}^{\prime} \mathrm{CO}_{2}$, respiratory frequency (fR), end-tidal (FE'Iso) and inspired fractions (FI'Iso) of 
Iso. The respiratory gas monitor was calibrated every 6 months by a General Electric (GE) authorized engineer using a GE proprietary calibrating gas cylinder connected to the monitor's sampling line. A Cardiocap II (Datex-Ohmeda, Finland) was used to continuously monitor the heart rate and arterial blood pressure and each variable was recorded at 5-minute intervals. A facial or transverse facial arterial catheter connected to a pressure transducer (Pressure Transducer DTX/Plus; Becton Dickinson) positioned and zeroed at the level of the sternal manubrium was used for invasive arterial blood pressure measurement. Once the first FE' Iso target $\left(\mathrm{ET}_{\text {target }}\right)$ was attained $(1.5 \%)$, the subsequent $\mathrm{ET}_{\text {target }}$ adjustments were based on clinical signs of anaesthesia depth. When the anaesthesia plane was either too light (brisk palpebral (blinking) reflex, presence of swallowing, tightening of neck muscles) or excessive (i.e. when the eye globe was central and the palpebral and/or the corneal reflex was absent), the $\mathrm{ET}_{\text {target }}$ Iso concentration was increased or decreased by $0.2 \%$, respectively. If the mean arterial pressure was lower than $70 \mathrm{~mm} \mathrm{Hg}$, dobutamine (Dobutamina Hospira; Hospira, Italy) was infused, starting at 0.6 $\mu \mathrm{g} \mathrm{kg}^{-1}$ minute ${ }^{-1}$, and adjusted every 10 minutes up to a maximum infusion rate of $1.2 \mu \mathrm{g}$ $\mathrm{kg}^{-1}$ minute ${ }^{-1}$. Mean arterial blood pressure was maintained between 70 and $90 \mathrm{mmHg}$ by adjusting the dobutamine infusion.

If the horses displayed nystagmus or resisted the ventilator, a ketamine bolus was administered $\left(0.1-0.2 \mathrm{mg} \mathrm{kg}^{-1}\right) \mathrm{IV}$, or thiopental $\left(0.5-1 \mathrm{mg} \mathrm{kg}^{-1}\right) \mathrm{IV}$ was injected if they moved. Morphine was administered IV (0.1 $\left.\mathrm{mg} \mathrm{kg}^{-1}\right)$; Morfina Cloridrato Molteni, Italy) 10-20 minutes before the end of surgery.

\subsubsection{Group I}

The infusion system comprised a laptop computer, which was connected to a recently 
calibrated respiratory gas monitor (Capnomac Ultima) for downloading and processing physiological data and to a syringe pump (Graseby 3500 Anaesthesia Pump; Smiths Medical International, United Kingdom) to control Iso infusion.

A 50-mL plastic syringe, filled with liquid Iso (Isoba; Schering-Plough, United Kingdom), was connected to the inspiratory branch $(3 \mathrm{~cm}$ diameter, $170 \mathrm{~cm}$ long) of a large-animal plastic breathing hose, near the inspiratory valve, via a fine-bore extension tubing (LectroSpiral Polyethylene Coiled I.V. Extension Set; Vygon, France) and an 18 G needle. To avoid any inadvertent drainage of liquid anaesthetic into the circuit, the syringe driver was positioned below the level of the injecting port. To ensure that liquid anaesthetic did not reach the patient, the injection point was below the level of the endotracheal tube. The hose was positioned in such a way as to maintain the needle in an absolutely vertical position during anaesthesia. A 2-cm diameter metallic plate, fixed within the hose at the opposite side of the $18 \mathrm{G}$ needle, was used for the collection of Iso drops. Respiratory gas was continuously sampled at the Y-piece by the gas monitor, and the sampled gas was then returned to the expiratory branch of the circuit after measuring. The laptop computer, which used a Windows XP operating system ran a customized Visual Basic $\mathrm{C}++$ program which required the operator to enter the horse's body weight (BW), the breathing circuit volume $\left(\mathrm{V}_{\mathrm{CIRC}}\right)$, the functional residual capacity $\left(\mathrm{V}_{\mathrm{FRC}}\right)$, the blood-gas partition coefficient $\left(\lambda_{\mathrm{B} / \mathrm{G}}\right)$, the cardiac output $(\mathrm{Q})$ and the desired end-tidal Iso concentration (ET target $)$. The program calculated the volume of Iso to be injected based on two equations.

Volatile agent $(\mathrm{mL})=\left[\left(\left(\mathrm{V}_{\mathrm{CIRC}}+\mathrm{V}_{\mathrm{FRC}}\right) \times \mathrm{ET}_{\text {target }}\right)+\left(\mathrm{ET}_{\text {target }} \times \lambda_{\mathrm{B} / \mathrm{G}} \times \mathrm{Qs}\right)\right] / 206$ 
Equation 1 was used to determine the prime dose, where $\mathrm{V}_{\mathrm{CIRC}}(28,600 \mathrm{~mL})$ was the total volume of the breathing system and ventilator, $\mathrm{V}_{\mathrm{FRC}}\left(22 \mathrm{~mL} \mathrm{~kg}^{-1}\right)$ was the functional

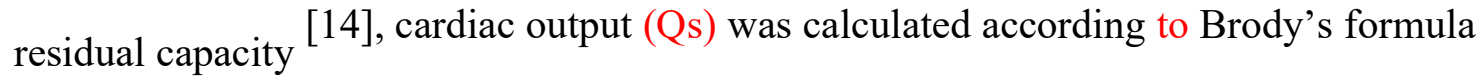
$\left(200 \times \mathrm{BW}^{0.75}\right)[15]$, and $\lambda_{\mathrm{B} / \mathrm{G}}(1.4)$ was the blood/gas partition coefficient.

Isoflurane $(\mathrm{mL})=\left[2 \times \lambda_{\mathrm{B} / \mathrm{G}} \times\left(200 \times \mathrm{BW}^{0.75}\right) \times\left(\mathrm{ET}_{\text {target }}-\mathrm{ET}_{\text {measured }}\right)+\left(\mathrm{FGF}-\left(\mathrm{BW}^{0.75} \times 0.07\right)\right.\right.$ $\left.\left.\mathrm{x} \mathrm{ET}_{\text {measured }}\right)\right] / 206$

In order to correct the difference between the measured and the targeted Iso concentrations, Equation 2 determined the volume of Iso to be administered every minute. The amount of volatile anaesthetic The amount of Iso flushed out by the FGF inflow every minute was computed in Equation 2. According to Kleiber [16], the basal oxygen uptake is determined to be $0.7 \times 10 \times \mathrm{BW}^{0.75}$, which is then reduced by $30 \%$, given that oxygen consumption decreases under anaesthesia [10]. The end-tidal Iso concentration, as a percentage of the barometric pressure measured by the respiratory monitor, yielded the value for FE'Iso ${ }_{\text {measured }}$<smiles>[Te][Te]</smiles>

The following data were displayed on the laptop monitor: the Iso infusion speed (mL hour ${ }^{1}$ ), the Iso volume to be next infused, the total infused Iso volume and $\mathrm{ET}_{\text {measured. }}$ Re-entry of the $\mathrm{ET}_{\text {target }}$ and FGF values was permitted at any stage during the procedure. The software also permitted the infusion to be paused or stopped at any time. 
Iso administration was initiated by the activation of the delivery infusion system. $\mathrm{An}_{\mathrm{ET}} \mathrm{T}_{\text {target }}$ of $1.5 \%$ was set and the prime dose delivered over a 2-minute period. $\mathrm{ET}_{\text {measured }}$ data, obtained from the gas analyser, were sent to the computer over a 1-minute period once the prime dose was delivered. Then, based on Equation 2, Iso was delivered as an infusion over 20 seconds, while taking account of the fact that the lowest end-tidal concentration of Iso received by the computer is $\mathrm{ET}_{\text {measured. }}$. Following complete infusion of the prime dose, the FGF of oxygen $(100 \%)$ was set at $4 \mathrm{~L}_{\text {minute }}{ }^{-1}$ until an inspired fraction of 0.7 was achieved, following which the rate was then reduced to $2 \mathrm{~L}_{\text {minute }}{ }^{-1}$. Any change in FGF in this group was double counted as FGF adjustment required because the anaesthetist not only had to modify the flowmeter but also to manually enter the new FGF into the system every time it was changed.

A VOC system (TEC 3; Ohmeda, Finland) was available for use if the anaesthetist discontinued the liquid Iso delivery for any reason.

\subsubsection{Group $V$}

Iso was delivered using a VOC vaporizer (TEC 4; Ohmeda, Finland) throughout the experimental study. The vaporizer was always refilled while in the same position, leaning against the edge of a horizontal surface delineated by red tape stripes. A bottle of Iso was connected to the vaporizer and positioned lower than the leaning plane such that, once the inlet valve was opened, all of the Iso decanted completely from the vaporizer into the bottle. Once the flow of Iso ceased, the inlet valve was closed and the bottle was disconnected. A prefilled bottle containing $50 \mathrm{~mL}$ of Iso was sequentially connected and again all the fluid was introduced into the vaporizer. The anaesthetist was free to use the 
preferred FGF rate and to adjust the vaporizer setting in order to reach and maintain the desired FE'Iso target of $1.5 \%$. When the depth of anaesthesia needed to be altered, the anaesthetist was again free to set the vaporizer and FGF as preferred. Similarly to group I, FE'Iso was varied by a $0.2 \%$ step: if the first FE'Iso target $(1.5 \%)$ was insufficient to provide an adequate anaesthetic plane, FE' Iso was raised to $1.7 \%$. Once the fraction of inspired oxygen was 0.7 and the plane of anaesthesia was stable (based on clinical signs), FGF was progressively reduced, aiming to set it to $2 \mathrm{~L}$ minute ${ }^{-1}$. Each variation in the vaporizer setting and FGF was recorded on the anaesthesia sheet. When the anaesthesia time reached 1 hour, the vaporizer in use was turned off, removed from the back bar and replaced with another prefilled Iso vaporizer (Tec 3 - Ohmeda). The amount of decanted Iso was measured with a 10-mL syringe under an extractor hood. The amount of consumed Iso was equal to $50 \mathrm{~mL}$ minus the decanted amount $(\mathrm{mL})$. In this group, the time from opening the vaporizer to reaching the target FE'Iso of $1.5 \%$ was measured.

\subsubsection{Recovery from anaesthesia}

To assist recovery from anaesthesia, the horses were sedated with xylazine $\left(0.3 \mathrm{mg} \mathrm{kg}{ }^{-1}\right.$; IV), while oxygen (15 L minute ${ }^{-1}$ ) was administered intranasally. The duration of lateral and sternal recumbency, the time of extubation and the total time to standing following disconnection of the anaesthetic circuit were noted. Descriptors of the horses' recoveries were used to assign a "recovery score" to each horse, using a scale ranging from 1 to 5 ,with 5 being the best (horse stood easily after one attempt during recovery from anaesthesia) and 1 being the worst (horse could not stand without sling support or sustained significant injury during recovery) [17].

\subsection{Statistical analysis}


Data were analysed using a commercially available statistical package (SAS 9.3, SAS Institute). Categorical variables were expressed as frequencies and percentages. The correlation between the linear variables - BW and consumed Iso expressed in millilitres was evaluated with Pearson's correlation coefficient. Continuous variables were checked for normal distribution by visual inspection of bar graphs, histograms and the ShapiroWilk test. Normally distributed variables were reported as the mean \pm the standard deviation (SD), whereas non-normally distributed variables were expressed as the median (range). Differences between non-normally distributed data were analysed with the MannWhitney test. Statistical significance was set at $P<0.05$. The data are presented as boxand-whisker plots, where the triangle represents the mean and the horizontal line the median, the box ends represent the interquartile range, and the upper and lower lines (or whiskers) represent the maximum and minimum values of data collected in each group.

\section{Results}

Twenty-five horses, classified as ASA I on physical examination, were enrolled in this study; one horse in group I was excluded because the computer malfunctioned during the anaesthetic procedure. As a consequence, 24 horses -7 intact males, 3 geldings and 14 intact females undergoing scheduled surgical procedures - were included in the study. Of these, 10 were assigned to group I and 14 to group V. Both groups were similar in terms of age, body mass and number of subjects anaesthetized as first of the day (Table 1). Horses belonging to group I underwent the following procedures: arthroscopy $(n=8)$, desmotomy $(\mathrm{n}=2)$, while, the group $\mathrm{V}$ procedures were: $\operatorname{arthroscopy}(\mathrm{n}=11)$, splint removal $(\mathrm{n}=1)$, cataract surgery $(\mathrm{n}=1)$, bone marrow collection and bone marrow concentrate aspirate 
injection $(n=1)$.

Gas leakage from the breathing system was less than $0.5 \mathrm{~L} \mathrm{~min}^{-1}$ at $25 \mathrm{cmH}_{2} \mathrm{O}$ when tested. Data on the amount of volatile anaesthetic consumed during the surgical procedure for one horse in group $\mathrm{V}$ were lost due to the aforementioned system crash.

Three horses in group I and two in group V received xylazine during premedication. Four horses in group I and five in group V received ketamine during maintenance of anaesthesia. Thiopentone was administered once to a horse in group I.

Dobutamine was administered to six out of $10(60 \%)$ and 10 out of $14(71.43 \%)$ horses, respectively, in groups I and $\mathrm{V}$.

At the beginning of the anaesthesia, the median FGF in group V was 8 (7-10), while the median setting of the vaporizer was $4(3.5-4)$. The median consumption of Iso, during the first hour of surgery, was significantly lower in group I compared with group V (20.5 (9.331.4) $\mathrm{mL}$ vs $29.1(21.5-43.0) \mathrm{mL}, P=0.003)$ (Figure 2 ), with a median consumption of volatile anaesthetic per kilogram of BW of $0.042(0.028-0.052)$ and $0.062(0.037-0.094)$ $\mathrm{mL} \mathrm{kg}^{-1}$, respectively, in groups I and $\mathrm{V},(P=0.009)$.

The median numbers of FE'Iso target variations during anaesthesia were the identical in the two groups $-1(0-3)$. The median numbers of total drug delivery adjustments required by the ETCLo-TCI system in group I and the vaporizer in group V in the first hour of anaesthesia were significantly different (group I 1.5 (0-3) vs group V 4 (3-9), $P=0.0002$ ) (Figure 3). No statistical difference was found between the two groups concerning the number of FGF adjustments required (group I 2 (1-7) vs group V 3 (2-7), $P=0.19$ ). A significant correlation between the total consumption of Iso in 1 hour and BW was observed in group I ( $\mathrm{R}^{2}=0.92$, Pearson's coefficient $0.96, P<0.001,95 \%$ CI 0.82 to 0.99$)$ 
(Figure 4), but not in group V $\left(\mathrm{R}^{2}=0.21\right.$, Pearson's coefficient $-0.46, P<0.001,95 \% \mathrm{CI}-$ 0.80 to 0.14$)$.

No significant difference was found in the time taken by both systems for volatile anaesthetic delivery to reach the end-tidal target (group I 8 (6-16) vs group V 10 (4-15) (P $=0.69)$.

There was no significant difference regarding the quality of the recovery phase between groups I and V, as assessed by the recovery scores. In group I, six horses received a recovery score of 5 , three of 4 and one of 3 , while, in group $\mathrm{V}$, nine horse received a recovery score of 5 , three of 4 and two of 3 .

\section{Discussion}

This is the first published study to compare an electronic anasthesia infuser system to deliver inhaled agents with-a what can be considered the "standard" in volatile agent delivery, the VOC system to maintain anaesthesia in horses This is the first published study to compare an electronic anaesthesia infuser system with a VOC system for the maintenance of anaesthesia in horses. Although, the ETCLo-TCI system is a low-budget prototype, made by connecting different pieces of equipment (anaesthetic monitor, a syringe driver and a personal computer) using simple software, this system has proven to provide some advantages over the VOC system. As is shown by the high correlation coefficient between the amount of Iso used to produce 1 hour of anaesthesia and horse weight, the ETCLo-TCI system produces quantitative volatile agent anaesthesia. Although quantitative anaesthesia is often associated, as shown in the literature, with the use of a closed-circuit breathing system [6], it has not previously been demonstrated for an ETCLo- 
TCI system. One advantage of the tested delivery system is that it is able to adapt the anaesthetic administered to the FGF set by the annesthetist, because the FGF is used in Equation (2) to caleulate the amount of Iso that has to be delivered One advantage of the tested delivery system is that it is able to adapt the anaesthetic administered to the FGF set by the anaesthetist as per Equation (2). In this study, it was decided to set the fraction of inspired oxygen $\left(\mathrm{FiO}_{2}\right)$ to a value of 0.7 to decrease the risk of hypoxemia. Horses under anaesthesia develop a high pulmonary shunt fraction; a supra-physiological $\mathrm{FiO}_{2}$ can increase arterial blood saturation [18], even though some drawbacks of this strategy have been reported [19]. Low flow anaesthesia may cause a build-up of unwanted gas, such as methane or acetone, within the breathing system. It has been suggested that, when using this technique, the operator should periodically raise the FGF in order to decrease the concentration of contaminants [20]. However, in this study, once the $\mathrm{FiO}_{2}$ had reached 0.7, the FGF was lowered to $2 \mathrm{~L}_{\text {minute }}{ }^{-1}$ as it was then easier to detect a gas leakage from the breathing system because of the emptying of the ventilating bellows, and this may have had a positive impact on reducing air pollution in the operating theatre.

The ETCLo-TCI system crashed because the software malfunctioned in one case out of 11. This would be an unacceptable failure rate for a device used in clinical anaesthesia. However, the delivery system tested in this study is a prototype and has a degree of instability and software issues that need to be resolved.

The amount of Iso consumed in group I was significantly lower than in group V. Given that a $250 \mathrm{~mL}$ bottle of Iso costs about 50 Euro, 2 Euro could be saved during the first hour of 
anaesthesia per horse anaesthetized. A greater saving, however, would occur if sevoflurane were used. Although, this whoth not produce a significant reduction in anesthesia costs, $m$ Monitoring the amount of inhaled agent consumed when performing quantitative anaesthesia may open new research fields. Under certain conditions, the uptake of volatile anaesthetics could theoretically be used to provide information on cardiac output [21]. Moreover, research into large-animal anaesthesia could be directed towards the design of inhalational anaesthesia work stations based on the technology used in this study, where anaesthetists simply set their targets (end-tidal agent and oxygen concentration), allowing the machine to calculate the exact amount of anaesthetic to deliver. The system would constantly monitor these values and automatically adjust the gas delivery and total flow to achieve and maintain the set target values. In this study, the large-animal circle breathing system was dismantled, cleaned and left to dry overnight once the last case of the day had recovered from anaesthesia. This ensured that the system did not contain any residual Iso at the beginning of the following day. The first case of the day may have required more anaesthetic, and, for this reason, the authors wanted to record the number of first-of-day cases anaesthetized in this study. Anaesthetic uptake from the lung by the blood also depends on cardiac output - the higher the output, the higher the uptake of anaesthetic by the blood. The substantial difference in cardiac output between groups I and V may have caused differences in the consumption of isoflurane, independent of the delivery system's performance. Nevertheless, the percentage of horses that received dobutamine during the maintenance of anaesthesia was similar in both groups. When a volatile agent is administered by a vaporizer at the beginning of 
anaesthesia, the anaesthetic concentration has to be significantly higher than the FE'Iso target, which should be adequate to maintain general anaesthesia. This is due to the fact that the anaesthetic requirement is very high in this phase because the large-animal circle breathing system has to be filled with the anaesthetic, and this is also the period when the anaesthetic uptake by the patient is at its highest. There are two methods to provide an elevated amount of volatile anaesthetic using a VOC vaporizer as the delivery system over a short time - flushing the breathing system with a high FGF containing a concentration of the volatile agent vapour close to the FE' of the volatile anaesthetic target and/or setting the vaporizer to deliver a high concentration of the drug. Once a horse is adequately

anaesthetized, the anaesthetist decreases the FGF and the percentage of volatile anaesthetic delivered by the vaporizer [22]. This entire process is time-consuming, requires repeated drug delivery adjustments and a considerable degree of expertise Delivering a volatile anaesthetic by direct injection as a liquid into the breathing system allows modulation of the delivery as desired. ETCLo-TCI involves a two-step administration mode. Initially, a loading dose is delivered to raise the FE'Iso to a target value (1.5\% in this study). When using a VOC vaporizer, the amount of wasted volatile anaesthetic is high at the beginning of anaesthesia and also rises every time the FE' target has to be varied. In the later part of a surgical procedure, anaesthesia tends to be more stable and variations in the FE'Iso target are generally less frequent. The difference in Iso consumption between groups I and V would have been less pronounced if the second hour of anaesthesia were also taken into account.

In order to avoid a potentially serious cardiovascular consequence, as previously reported [6], the loading dose was administered over 2 minutes. This can explain why there was no 
significant difference in the time taken by both systems to reach the FE'Iso target. However, this lack of difference should be considered as a desirable safety feature of ETCLo-TCI. The second administration modality delivers on a minute-by-minute basis the amount of Iso that is necessary to raise the FE'Iso to the target. Therefore, the Iso delivery is completely independent of the FGF as the carrier, which can be set to achieve the desired $\mathrm{FiO}_{2}$, as it was in this study. The number of FE'Iso target changes during the surgical procedures was similar in both groups; nevertheless, the required number of drug delivery adjustments differed significantly between both groups. The anaesthesiologist's vigilance has been shown to decrease as the number of tasks increases [23, 24]. However, the use of the ETCLo-TCI system may reduce the time required by the anaesthetist to provide an adequate anaesthetic plane compared with systems that use a VOC vaporizer. In order to minimize the consumption of Iso in group $\mathrm{V}$ once the plane of anaesthesia was stable, the anaesthetist reduced the FGF by up to $2 \mathrm{~L}$ minute ${ }^{-1}$. This reduction in FGF may have forced the operator to modify the vaporizer setting more often than normal to maintain the desired FE'Iso, thus potentially introducing a bias into this study.

This study did not find differences in the number of FGF changes between the two groups. However, any changes in FGF were double counted in group I. This decision was taken for the reason expressed above. A more innovative version of this delivery system using an electronic flowmeter could avoid the manual input of the FGF value into the system - such a system would acquire the FGF value directly from an electronic flowmeter. Considering the non-relevance of FGF regarding anaesthetic administration when using the ETCLo-TCI system, an upgraded version might just require the simple entry of the $\mathrm{FiO}_{2}$ target; the FGF could be automatically regulated by a closed-loop feedback system to achieve this target. 
The anaesthetist was left free to premedicate horses with xylazine or romifidine. The shorter duration of action of xylazine may have caused the need for a higher FE'Iso in horses premedicated with this drug, increasing the number of drug delivery adjustments required compared with subjects receiving romifidine.

A greater number of horses in group V compared with group I had a recovery score of 5; however, it is very unlikely that the volatile agent delivery system would have played any role in the recovery quality from anaesthesia - this was influenced by other factors [17]. Erroneous high readings of anaesthetic agent concentrations can be caused by interfering with the anaesthetic gas analysis of methane $[25,26]$, which can accumulate in rebreathing systems in anaesthetized horses [27]. Methane absorbs infrared light at a wavelength of 3.3 $\mu \mathrm{m}$, which is the operating basis behind the use of infrared absorption technology to measure the concentration of volatile anaesthetic agents in a respiratory gas sample using a Capnomac Ultima gas analyser, as in this study. Dujardin et al. [28] found that methane builds up within the breathing system when a FGF as low as 1.5-2 $\mathrm{L}$ minute $^{-1}$ is delivered, causing an associated error in the volatile anaesthetic concentration reading. Therefore, the FGF was kept low enough to potentially cause the build-up of methane within the breathing system in both groups. FGF was lowered earlier in group V than in group I. When the $\mathrm{ET}_{\text {target }}$ was raised in group $\mathrm{V}$, the FGF may have also been raised. However, considering the number of $\mathrm{ET}_{\text {target }}$ variations, the $\mathrm{ET}_{\text {target }}$ value used during maintenance of anaesthesia, the number of ketamine and/or thiopentone boluses administered and the fact that the number of subjects treated with dobutamine did not differ, it is unlikely that possible differences in FGF in some subjects would have jeopardized the study results. Koenig et al. [29] found $\mathrm{ETCO}_{2}$ to be a poor predictor of the partial pressure of $\mathrm{CO}_{2}\left(\mathrm{PaCO}_{2}\right)$ in arterial 
blood in anaesthetized horses. Physiological dead space and intrapulmonary shunting can significantly affect the alveolar-arterial gradient of gases and vapours, introducing a variable source of error to the volatile anaesthetic end-tidal reading. As explained in the Materials and Methods section, the $\mathrm{ET}_{\text {target }}$ was adjusted during anaesthesia maintenance based on clinical signs of depth of anaesthesia, regardless of the volatile anaesthetic Eendtidal reading displayed on the monitor. Based on all these facts, the authors consider it improbable that methane accumulation accounted for the inter-group differences. Eonsidering the high $\mathrm{FGF}$ required to reach the desired $\mathrm{FiO}_{2}$, the fat that data were only collected for 1 hour of annesthesia and the similar annesthetic conditions for both groups, it is improbable that methane acemmulation had either an effect on group comparison or on anaesthesia quality. The difference between the $\mathrm{ET}_{\text {medwed }}$ and the Iso arterial blood eoncentration may well be increased by methane acemmulated in the breathing system. Koenig et al. [23] found ETCO $\left(\mathrm{PaCO}_{z}\right)$ in arterial blood in anaesthetized horses. Nevertheless, mechanically ventilated healthy horses have shown a miner mean difference in the alveolar-arterial pressure of $\mathrm{CO}_{z^{-}}$ $[23,24]$.

\section{Conclusions}

The use of electronic devices for anaesthetic dosing offers the opportunity to achieve precise dosing, while requiring less drug dosage and fewer adjustments by the anaesthetist. The ETClo-TCI system produces quantitative volatile agent anaesthesia, thus providing a simple means for anaesthetic uptake monitoring. Further studies should be directed towards 
developing this volatile anaesthetic delivery system by adding an electronically controlled flowmeter.

\section{Acknowledgements}

The authors wish to thank Dr. Andrea Bertamini and Dr. Oscar Bertamini for helping to realize this study.

\section{Reference:}

[1] Steinbereithner K. What does quantitative anaesthesia mean? In: Van Ackern K, Frankenberger H, Konecny E SK, editors. Quantitative anaesthesia. 1th ed., Berlin: Springer-Verlag; 1989, p. 3-15.

[2] Baum J. Clinical applications of low flow and closed circuit anesthesia. Acta Anaesthesiol Belg 1990;41: 239-47

[3] Verkaaik AP, Van Dijk G. High flow closed circuit anaesthesia. Anaesth Intensive Care 1994;22:426-34.

[4] Westenskow DR, Jordan WS, Hayes JK. Uptake of enflurane: a study of the variability between patients. Br J Anaesth 1983;55:595-601.

[5] Zbinden AM, Frei F, Westenskow DR, Thomson DA. Control of end-tidal halothane concentration. Part B: Verification in dogs. Br J Anaesth 1986;58:563-71.

[6] Olson KN, Klein LV, Nann LE, Soma LR. Closed-circuit liquid injection isoflurane anesthesia in the horse. Vet Surg n.d.;22:73-8.

[7] Moens Y. Introduction to the quantitative technique of closed circuit anesthesia in dogs. Vet Surg 1988;17:73-8.

[8] Boller M, Moens Y, Kastner LE, Soma SBN, Bettschart-Wolfensberger R. Closed system anaesthesia in dogs using liquid sevofluorane injection; evaluation of the square-root-of-time model and the influence of $\mathrm{CO} 2$ absorbent. Vet Anaesth Analg 2005;32:168-77.

[9] el-Attar AM. Guided isoflurane injection in a totally closed circuit. Anaesthesia 1991;46:1059-63.

[10] Struys MMRF, Kalmar AF, De Baerdemaeker LEC, Mortier EP, Rolly G, Manigel J, et al. Time course of inhaled anaesthetic drug delivery using a new multifunctional 
closed-circuit anaesthesia ventilator. In vitro comparison with a classical anaesthesia machine. Br J Anaesth 2005;94:306-17.

[11] Potdar MP, Kamat LL, Save MP. Cost efficiency of target-controlled inhalational anesthesia. J Anaesthesiol Clin Pharmacol 2014;30:222-7.

[12] Franci P, Bertamini A, Bertamini O, Pilla T, Busetto R. Clinical evaluation of an end-tidal target-controlled infusion closed-loop system for isoflurane administration in horses undergoing surgical procedures. Vet J 2012;192:206-11.

[13] Lowe HJ, Ernst EA. The Quantitative Practice of Anesthesia: use of closed circuit. Baltimore: William and Wilkins; 1981, p. 19.

[14] McDonell W, Hall L. Functional residual capacity in conscious and anaesthetized horses. Br J Anaesth 1974;46:802.

[15] Brody S. Bioenergetics and Growth. New York: Reinhold Publishing Corporation; 1945 , p. 368-73.

[16] Kleiber M. Body size and metabolic rate. Physiol Rev 1947;27:511-39.

[17] Young SS, Taylor PM. Factors influencing the outcome of equine anaesthesia: a review of 1,314 cases. Equine Vet J 1993;25:147-151.

[18] Marntell S, Nyman G, Hedenstierna G. High inspired oxygen concentrations increase intrapulmonary shunt in anaesthetized horses. Vet Anaesth Analg 2005;32:338-47.

[19] Eisner MD, Balmes JR. Indoor and outdoor air pollution. In: Mason R, Broaddus C, Murray J, editors. Murray \& Nadel's textbook of respiratory medicine 2. 2th ed. Philadelphia: Saunders Elsevier; 2005, p. 1601-18.

[20] Baum JA. Low-flow anesthesia: theory, practice, technical preconditions, advantages, and foreign gas accumulation. J Anesth 1999;13:166-74.

[21] Kennedy RR, Baker AB. The effect of cardiac output changes on end-expired volatile anaesthetic concentrations: a theoretical study. Anaesthesia 2001;56:103440 .

[22] Doherty T, Greene SA. Anesthetic Circuits. In: Greene SA, editor. Veterinary anesthesia and pain management secrets. 1th ed., Philadelphia: Hanley \& Belfus; 2002, p. 65-9.

[23] Weinger MB, Herndon OW, Gaba DM. The effect of electronic record keeping and transesophageal echocardiography on task distribution, workload, and vigilance during cardiac anesthesia. Anesthesiology 1997;87:144-55.

[24] Weinger MB, Reddy SB, Slagle JM. Multiple measures of anesthesia workload during teaching and nonteaching cases. Anesth Analg 2004;98:1419-25.

[25] Moens YP, Gootjes P. The influence of methane on the infrared measurement of anaesthetic vapour concentration. Anaesthesia 1993;48:270.

[26] Rolly G, Versichelen LF, Mortier E. Methane accumulation during closed-circuit anesthesia. Anesth Analg 1994;79:545-7. 
[27] Morita S, Latta W, Hambro K, Snider MT. Accumulation of methane, acetone, and nitrogen in the inspired gas during closed-circuit anesthesia. Anesth Analg 1985;64:343-7.

[28] Dujardin CLL, Gootjes P, Moens Y. Isoflurane measurement error using short wavelength infrared techniques in horses: influence of fresh flow and pre-anaesthetic food deprivation. Vet Anaesth Analg 2005;32:101-6.

[29] Koenig J, McDonell W, Valverde A. Accuracy of pulse oximetry and capnography in healthy and compromised horses during spontaneous and controlled ventilation. Can J Vet Res 2003;67:169-74. 
Fig. 1: The end-tidal closed-loop target-controlled infusion device (ETCLo-TCI) system. In this system isoflurane is directly injected into the breathing system by a closed-loop system, which comprises a laptop computer (with dedicated software), a computer-controlled syringe driver (loaded with liquid isoflurane) connected to the inspiratory arm of a largeanimal circle breathing system and a respiratory gas monitor.

Fig. 2: Number of isoflurane delivery device setting variations in the first hour of anaesthesia recorded in 10 horses using an ETCLo-TCI delivery system (group I) and in 14 horses using a VOC vaporizer (group V). Data collected are statistically significant $(P<$ $0.05)$ and presented as box-and-whisker plots.

Fig. 3: Median consumption of isoflurane $(\mathrm{mL})$ in the first hour of anaesthesia recorded in 10 horses using an ETCLo-TCI delivery system (group I) and in 14 horses using a VOC vaporizer (group V). Data collected are statistically significant $(P<0.05)$ and presented as box-and-whisker plots.

Fig. 4: Significant correlation (Pearson's coefficient $0.96, P<0.001,95 \%$ CI 0.82 to 0.99 ) between the total consumption of isoflurane $(\mathrm{mL})$ in the first hour of anaesthesia and the body weight in 10 horses using an ETCLo-TCI delivery system (group I). Data are presented in a scatter plot with regression line. 